\title{
Silviahemmet-Konzept unter der Lupe
}

\section{Studie: Demenzstation hat sich bewährt}

") Die Versorgung von Menschen mit Demenz im Akutkrankenhaus ist vielerorts ein ungelöstes Problem. Nicht selten verschlechtert sich die Demenzsymptomatik im ungewohnten Klinikumfeld. Dass es auch anders geht, beweist das Malteser Krankenhaus St. Hildegardis in Köln.

Über drei Jahre haben Forscher des Deutschen Instituts für angewandte Pflegeforschung (DIP) die Versorgung von 400 Patienten auf der ,Station Silvia" des St. Hildegardis Krankenhauses begleitet. Mobilität, geistige Fähigkeiten und Alltagskompetenz der Patienten verbesserten sich während des Aufenthaltes dort deutlich, wie die Evaluationsstudie des DIP ergab. Die Station ist als „special care unit“ auf die Bedürfnisse von Menschen mit Demenz zugeschnitten.

\section{Repräsentative Umfrage \\ Roboter für Lasten}

॥ Dem Einsatz technischer Hilfsmittel wie Pflegerobotern würden $48 \%$ der Deutschen zustimmen, sollten sie als Pflegebedürftige zu Hause gepflegt werden. Das ergibt eine Umfrage von Forsa im Auftrag des Verbraucherzentrale Bundesverbands. Mit 68\% Zustimmung - Mehrfachnennungen waren möglich - könnten sie sich am ehesten den Einsatz von Pflegerobotern zur Hilfe beim Heben und Umlagern des zu pflegenden Menschen vorstellen. Mit 36\% Zustimmung findet sich auf Platz zwei der Einsatz zur schnellen Hilfe nach Stürzen, gefolgt von der Hilfe bei der Medikamenteneinnahme mit 32\%. Jeweils 25\% Zustimmung würden Pflegeroboter erfahren, wenn sie zur Hilfe beim Essen oder bei alltäglichen Pflegeaufgaben wie Waschen, Baden oder dem Toilettengang eingesetzt würden. 23\% der Bevölkerung ab 18 Jahre könnte sie sich sogar zur Beschäftigung und als Gesprächspartner vorstellen.

(maw)
Aus Sicht des DIP setzt das Konzept in einem weiteren Punkt Maßstäbe: Die überwiegend hochbetagten Patienten wurden nicht am Bett fixiert. In vielen Krankenhäusern sei das noch der Fall, um die Patienten am Weglaufen zu hindern. „Fixierungen sind vermeidbar und keine natürliche Begleiterscheinung einer Krankenhausbehandlung", stellte Prof. Michael Isfort, stellvertretender Vorstandsvorsitzender des DIP, fest.

Auf der Station wurden Ansätze des schwedischen Silviahemmet-Konzepts umgesetzt, um eine Umgebung zu schaffen, in der Menschen mit Demenz optimal betreut werden können. Sie zeichnet sich durch eine übersichtliche Gestaltung aus und bietet mit Küche und sowie Tages- und Speiseraum eine Art familiären Zusammenlebens. Ärzte, Pflegepersonal und Therapeuten der

\section{Rating Report}

\section{Heimen geht es gut}

" Pflegeheime stehen zurzeit wirtschaftlich besser da als Kliniken. Nur bei wenigen ist die Insolvenzgefahr groß, eine Minderheit schreibt Verluste. $\mathrm{Zu}$ diesem Ergebnis kommt der „Pflegeheim Rating Report 2017“, der auf Analysen für das Jahr 2015 beruht. Als Problem sehen die Autoren die zunehmende Personalknappheit bei steigendem Personalbedarf. Der Bericht geht vom Anstieg der Zahl der Pflegebedürftigen von 2,9 Millionen 2015 auf 4,1 Millionen 2030 aus: „Damit verbunden ist ein zusätzlicher Bedarf von voraussichtlich 271.000 stationären Pflegeplätzen im Jahr 2030.“ Das erfordere Investitionen in Höhe von 53 bis 85 Milliarden Euro. Da vor allem privates Kapital benötigt werde, plädieren die Autoren für eine möglichst schlanke Gesetzgebung für Heime. Der deutsche Pflegemarkt sei noch sehr kleinteilig, die Bildung großer Verbünde in großem Maße möglich.

\section{DRK: Erstmals Frau an der Spitze Gerda Hasselfeldt zur Präsidentin gewählt}

" Einstimmig wählte die DRK-Bundesversammlung die frühere Bundesministerin und Bundestagsabgeordnete Gerda Hasselfeldt zur Präsidentin des Deutschen Roten Kreuzes. Sie folgt damit auf Dr. Rudolf Seiters, der nach 14 Jahren aus dem Amt scheidet, dem DRK aber weiterhin als Ehrenpräsident verbunden bleibt.

„Es ist mir eine Herzensangelegenheit, Menschen in Not zu helfen, ob in Deutschland oder in den vielen Krisenund Katastrophenregionen der Welt [...] Und auch wir in Deutschland müssen uns bei der Notfallversorgung der Bevölkerung auf neue Herausforderungen einstellen“, sagte Hasselfeldt.

Bundesinnenminister Dr. Thomas de Maizière würdigte Hasselfeldt als ,herausragende Persönlichkeit des öffentlichen Lebens" und das DRK als einen wichtigen Partner im Bevölkerungsschutz und bei Katastrophenfällen. 